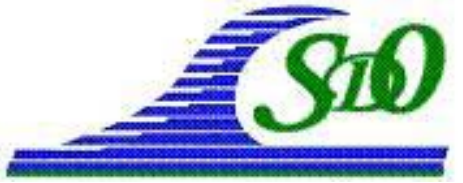

\title{
Gestion souple du littoral en harmonie avec la nature
}

\section{Nicholas GRUNNET ${ }^{1}$}

\author{
1. DHI, Agern Allé 5, 2970 Hørsholm, Danemark. \\ ngr@dhigroup.com
}

\begin{abstract}
Résumé :
L'art de développer des projets d'aménagement dans la zone littorale consiste en l'intégration des possibilités présentées par l'environnement marin avec les contraintes du projet. L'élément le plus attractif dans l'aménagement et la réhabilitation des zones littorales est souvent une plage artificielle. Pour la réussite d'un projet d'aménagement de plage artificielle, il est impératif d'inclure les aspects hydrauliques, côtiers et environnementaux dès le début du planning du projet. Le design de ces éléments doit suivre des "lois d'équilibre sédimentaire" qui imposent certaines restrictions sur le design. Les éléments les plus importants à observer sont les suivants : la plage rechargée devrait être exposée à la houle, orientée vers la direction prédominante de la houle pour être stable et soutenue par des ouvrages latéraux afin d'éviter des fuites de sédiments. Le sable devrait être moyen et bien trié avec un minimum de contenu de matériaux fins, grossiers et organiques. Les ouvrages côtiers adjacents aux plages devraient être aménagés de façon à ne pas générer des courants dangereux.
\end{abstract}

\section{Mots-clés :}

Gestion du littoral - Méthode souple - Protection côtière - Rechargement de plage

\section{Introduction}

Les techniques de défense contre la mer ont beaucoup évolué dans le temps. Les méthodes rigides furent les premières méthodes appliquées dans le domaine de la protection des côtes. Les méthodes rigides consistent à mettre en place des structures solides telles que perrés, épis et brise-lames dont l'action est de maintenir le trait de côte. Les dernières décennies ont vu apparaître des méthodes souples d'aménagement du littoral. Les méthodes souples telles que le rechargement de plage, la reconstitution des dunes et les systèmes de drainage de plage consistent à composer avec le milieu. Aujourd'hui des méthodes combinées sont de plus en plus fréquemment utilisées. Elles combinent méthodes souples et méthodes rigides. De manière générale, l'élément le plus attractif dans l'aménagement et la réhabilitation des zones littorales est souvent une plage artificielle. Afin de minimiser les coûts d'entretien des plages artificielles, il est souvent nécessaire de réaligner l'orientation de ces plages par rapport à l'orientation naturelle du site d'intérêt. Ceci nécessite typiquement la réalisation d'une structure côtière afin de stabiliser la plage. Ainsi, dans le contexte de projet d'aménagement du littoral, l'objectif principal s'est déplacé de la simple protection côtière vers une action 


\section{Thème 4 - Vulnérabilité du littoral}

plus intégrée à l'environnent naturel avec la prise en compte de valeurs esthétiques et récréatives.

L'art de développer des projets d'aménagement dans la zone littorale consiste en l'utilisation des possibilités d'un site spécifique pour le bénéfice du projet lui-même, c'est-à-dire d'intégrer les possibilités présentées par l'environnement marin avec les contraintes du projet. L'art est de percevoir les forces marines telles que les vagues et la marée comme des opportunités externes afin de maintenir des plages artificielles de bonne qualité contrairement à l'approche traditionnelle qui consiste à percevoir ces forces externes comme des générateurs de problèmes contre lesquelles une protection côtière est nécessaire.

Cette communication présente les caractéristiques des plages naturelles, des principes de design pour des plages artificielles notamment leur exposition à la houle et à la marée, du profil et l'alignement de la plage artificielle, et la nature des matériaux apte au rechargement ainsi que les structures côtières souvent nécessaires pour assurer la stabilité des plages artificielles. Cette communication présente également des exemples d'aménagements axés sur une gestion souple du littoral, qui ont été réalisés par la suite dans différents sites à travers le monde, notamment en France.

\section{Caractéristiques des plages naturelles}

Les plages attractives et récréatives sont caractérisées par leur exposition à une houle et à un régime tidal modérés, par de l'eau propre et transparente, par l'absence de plateaux rocheux, par du sable moyen bien trié et par un minimum de débris naturels et artificiels. Un exemple d'une telle plage naturelle attractive est illustré sur la figure 1. Bien que la composition et la couleur du sable peut varier d'un site à l'autre, les plages exposées ont une apparence sableuse et propre due à la houle qui empêche la déposition de matériaux fins et de matière organique. Néanmoins il existe beaucoup de plages sur des côtes ou la teneur en matières en suspension est élevée, au moins durant la saison des pluies et/ou durant des conditions énergétiques. Ceci est par exemple le cas sur la côte Ouest de la Malaisie et sur la totalité des plages du Sri Lanka. Ces plages aussi restent propres et sableuses parce qu'elles aussi sont exposées à la houle.

Clairement, le principal danger lié à l'aménagement d'une plage artificielle attractive est lié au manque d'exposition à la houle. Le manque d'exposition à la houle va permettre la déposition de matériaux fins sur les petits-fonds et l'estran, et cela même dans les cas où les plages ont été créées à partir de sable propre. Au fil du temps, la totalité de l'estran sera couvert de sédiments vaseux (voir figure 1 droite). Bien que cela résulte de mécanismes naturels, cela représente la raison principale de la mauvaise qualité des plages dans des environnements protégés. De telles plages sont généralement peu attractives. 

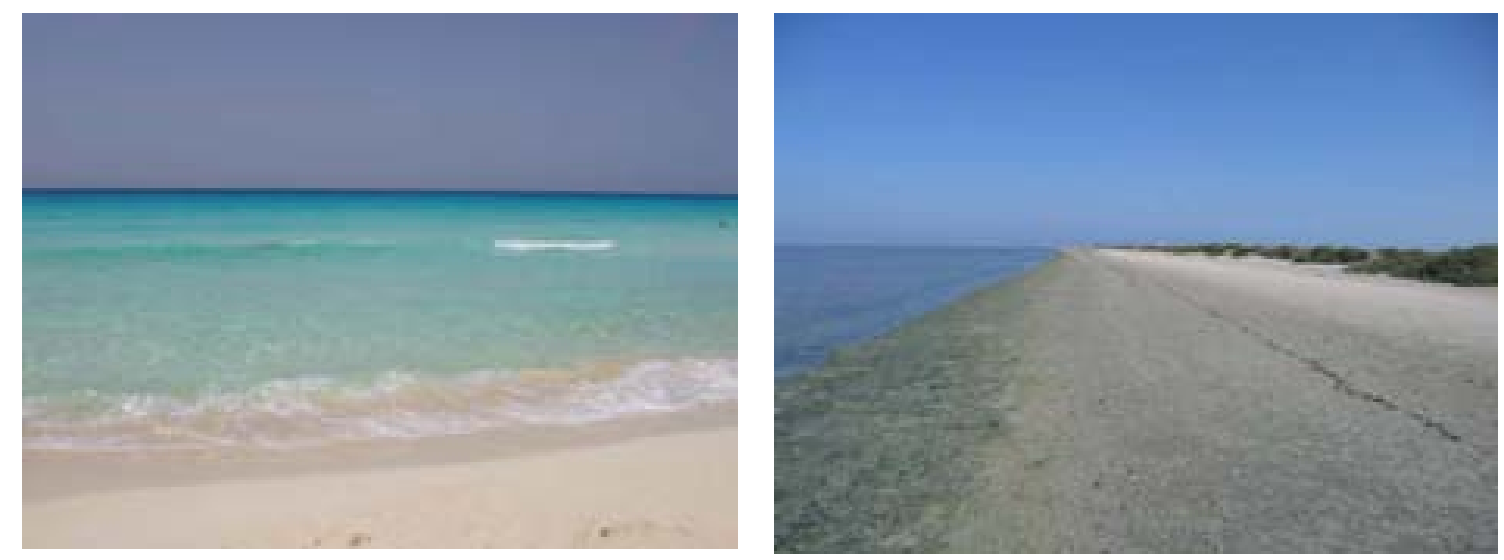

Figure 1. Plage bien exposée à la houle (gauche) sur la côte méditerranéenne en Egypte et moins exposée à la houle (droite) dans une lagune aux Emirats Arabes Unis.

\section{Principes de design pour plages artificielles}

L'élément le plus important dans beaucoup de projets d'aménagement du littoral est une plage sableuse attractive. Une plage artificielle consiste en la réalisation d'une nouvelle plage par apport externe de sable. Les principes de design pour assurer une plage récréative de bonne qualité sont donnés ci-dessous.

\subsection{Exposition à la houle}

La bonne qualité d'une plage est assurée par son exposition à la houle. Néanmoins, une plage récréative ne devrait pas être trop exposée puisque cela pourrait mettre en danger la sécurité des baigneurs. Il y a donc deux exigences opposées : un certain degré d'exposition afin d'assurer une plage sableuse, en revanche l'exposition ne devrait pas être trop importante afin d'assurer la baignade dans de bonnes conditions de sécurité.

Il doit être précisé que même pour des régions très énergétiques, une éventuelle destruction de plage en cas de tempête correspond à une redistribution du sable dans le profil si l'alignement de la plage est en équilibre (voir section 3.1), ainsi il n'y a pas de hauteur de houle à ne pas dépasser pour un site rechargé.

En vue de préserver une plage sableuse attractive, l'exposition à la houle devrait correspondre à une fréquence de dépassement de l'ordre de 12 heures par an pour une hauteur significative $\left(H_{\mathrm{s}, 12 \mathrm{~h} / \mathrm{an}}\right)$ supérieure à $1 \mathrm{~m}$. Le mouvement de sable continu par l'action de la houle durant des conditions énergétiques est le processus qui maintient naturellement une belle plage sableuse en y empêchant la déposition de matériaux fins, généralement présents dans les zones littorales. C'est également l'exposition à la houle qui empêche l'apparition de végétation marine sur l'estran.

Il n'existe pas de critères internationalement admis qui mettent en relation la hauteur de la houle avec des conditions favorables à la baignade. Ces conditions sont liées à l'occurrence et au type de déferlement de houle ainsi que les courants engendrés par la houle dans la zone de déferlement. 


\section{Thème 4 - Vulnérabilité du littoral}

Le déferlement glissant est souvent associé avec la formation de barres et courants de baïnes qui peuvent entraîner adultes et enfants en eau profonde. Cette situation est typique par vents forts et conditions tempétueuses sur des côtes (océaniques) sableuses. Le déferlement plongeant est dangereux due à sa violence qui peut s'abattre sur des baigneurs. Celui-ci est fréquent sur des côtes océaniques avec conditions de houle modérées et du sable relativement grossier. Ainsi, les côtes océaniques sont les plus dangereuses. Toutefois, si une valeur supérieure de la houle devrait être recommandée, un critère estimé serait de l'ordre de $H_{\mathrm{s}}<0,8-1,2 \mathrm{~m}$ durant la saison de baignade. La limite inférieure est valable pour des houles de longues périodes et la limite supérieure pour des houles à forte cambrure (mer de vent). Cela implique que des mesures protectrices, sous forme d'ouvrages de protection côtière spécialement conçus, sont nécessaires si un site est plus exposé que le critère indiqué.

Des courants générés par la présence d'ouvrages de protection côtière peuvent également être très dangereux. Ce danger est particulièrement accentué durant des événements énergétiques puisque la houle est atténuée à l'abri de l'ouvrage, mais en même temps des courants forts sont engendrés sous forme de circulations tourbillonnaires capables d'entrainer des baigneurs en eau profonde. De telles plages aménagées offrant une zone partiellement abritée génèrent une fausse sensation de sécurité et de tels aménagements devraient être évités.

Une plage récréative devrait avoir un profil actif jusqu'à une profondeur d'environ $2 \mathrm{~m}$ par rapport au niveau de basse mer afin de pouvoir maintenir des petits-fonds sableux sur la totalité de l'estran fréquentée par les baigneurs. En termes de hauteur de houle, une profondeur active de l'ordre de $2 \mathrm{~m}$ correspond à $H_{\mathrm{s}, 12 \mathrm{~h} / \mathrm{an}} \geq 1,0 \mathrm{~m}$.

Si le profil naturel ne permet pas que ces critères soient satisfaits suite à une pente trop faible de la plage, deux solutions sont possibles: la plage est transposée vers le large ou le profil existant est dragué pour accommoder une pente plus forte.

\subsection{Exposition à la marée}

Un marnage et une surcote élargissent une plage. Toutefois une terrasse tidale peut se développer si le marnage de vive-eau moyenne est bien plus important que la moyenne annuelle de la hauteur de la houle au point de déferlement $H_{\mathrm{b}}$. Un marnage élevé peut également présenter un danger aux baigneurs. Conséquemment, une plage récréative de bonne qualité est normalement caractérisée par un régime micro à mésotidal avec un marnage inférieur à environ $1,5 \mathrm{~m}$.

\subsection{Alignement et profil d'une plage artificielle}

L'alignement d'une plage artificielle devrait être stable (horizontalement) afin d'assurer un minimum d'entretien. Cela implique que l'orientation de la plage devrait être perpendiculaire à la direction prédominante de la houle, selon son orientation d'équilibre donc, correspondant à une dérive littorale nette nulle. Ceci nécessite souvent 


\section{XI $I^{\text {èmes }}$ Journées Nationales Génie Côtier - Génie Civil Les Sables d'Olonne, 22-25 juin 2010}

des ouvrages pour stabiliser la plage dans une orientation différente de l'orientation naturelle du site rechargé.

Sur une plage exposée avec une incidence oblique de la houle, les ouvrages soutenant une plage artificielle devraient satisfaire les conditions suivantes :

- Assurer un support latéral de la plage artificielle de façon à ne pas avoir de fuite de sédiments hors du site rechargé.

- Assurer une protection partielle contre l'action de la houle.

- Les courants dangereux près de la plage ne doivent pas être présents.

- Les ouvrages devraient être profilés selon les lignes de courants afin de minimiser l'échouage de débris flottants.

- Tous les ouvrages de protection côtière devraient avoir une fonction récréative.

Un profil de plage doit être stable et doit ainsi être réalisé selon son profil d'équilibre. Une plage évolue vers son équilibre dans la zone active du littoral. La forme du profil dépend principalement des caractéristiques granulométriques du sable. La forme en équilibre, selon le profil de Dean (DEAN, 2003), suit la forme $d=A x^{2 / 3}$ où $d(\mathrm{~m})$ est la profondeur à la distance $x(\mathrm{~m})$ du trait de côte. $A$ est la constante de Dean qui dépend de la granulométrie du sable selon le tableau 1.

Le concept de profil d'équilibre est uniquement valable dans la zone active du littoral, jusqu'à une profondeur $d_{1}$. De manière pratique, $d_{1} \sim 2 H_{\mathrm{s}, 12 \mathrm{~h} / a n}$ peut être admis pour une mer de vent.

Tableau 1. Corrélation entre le diamètre moyen $d_{50}$ en mm et la constante A dans l'équation du profile d'équilibre de Dean; pour sable de plage typique.

\begin{tabular}{lllll}
\hline $\boldsymbol{d}_{\boldsymbol{5 0}}$ & $\mathbf{0 . 2 0}$ & $\mathbf{0 . 2 5}$ & $\mathbf{0 . 3 0}$ & $\mathbf{0 . 5 0}$ \\
\hline $\boldsymbol{A}$ & 0.080 & 0.092 & 0.103 & 0.132 \\
\hline
\end{tabular}

\subsection{Nature des matériaux apte au rechargement de plage}

Les matériaux de rechargement devraient satisfaire les critères suivants afin d'obtenir une bonne qualité de plage récréative :

- Les caractéristiques du sable rechargé devraient être semblables à celles du sable local, toutefois légèrement plus grossier.

- Le sable devrait être moyen avec un diamètre compris entre $0,25 \mathrm{~mm}<d_{50}<0,50 \mathrm{~mm}$, préférablement plus grossier que $0,30 \mathrm{~mm}$ afin de minimiser les pertes dues au transport éolien.

- Le contenu de sable fin devrait être moins de 1-2\%.

- Le contenu de sable grossier et de coquillage devrait être moins de 3\%.

- Aucun contenu de matière organique.

Si le contenu de sable fin est trop important, la perméabilité est faible et le drainage de la plage sera lent durant la marée descendante. Cela implique que la plage sera 
constamment humide. Ceci est particulièrement important pour les plages artificielles aménagées dans des sites abrités, puisqu'il n'y a pas suffisamment d'exposition à la houle pour transporter les matériaux fins hors de l'estran. L'aspect vaseux résultant favorise également la croissance d'algues sur la plage.

La combinaison de manque d'exposition à la houle et de matière organique peut favoriser des conditions anoxiques qui engendrent la formation de sulphide d'hydrogène, entrainant une mauvaise odeur et une couleur noirâtre du sable (voir figure 2). Le critère du faible contenu de sable grossier est important pour la qualité du sable surfacique (voir figure 3) puisque la fraction de sable fin sera la première à être transportée par l'action de la houle vers les petits-fonds. Comme indiqué par les exemples des figures 2 et 3, l'utilisation de sable approprié est très importante pour la création de plages artificielles récréatives.
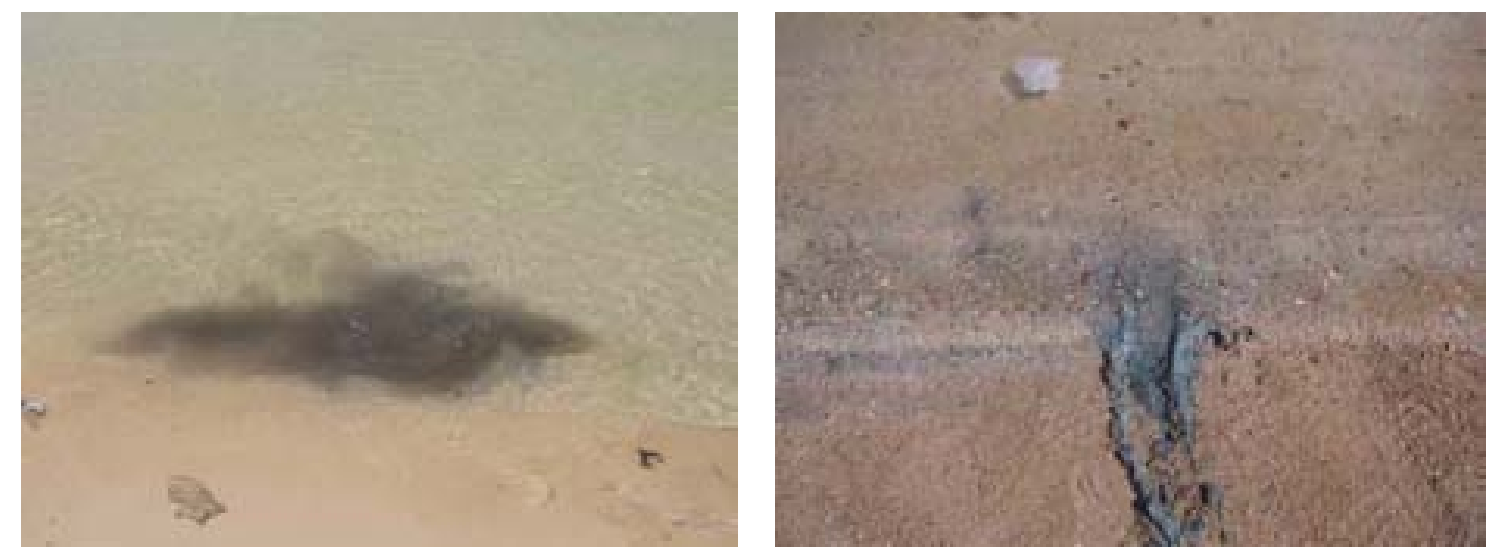

Figure 2. Conditions anoxiques et formation de sulphide d'hydrogène sur des plages artificielles dans des sites protégés : lagune artificielle dans la Mer Rouge (gauche) et plage artificielle dans une lagune sur la cote méditerranéenne en Egypte (droite).
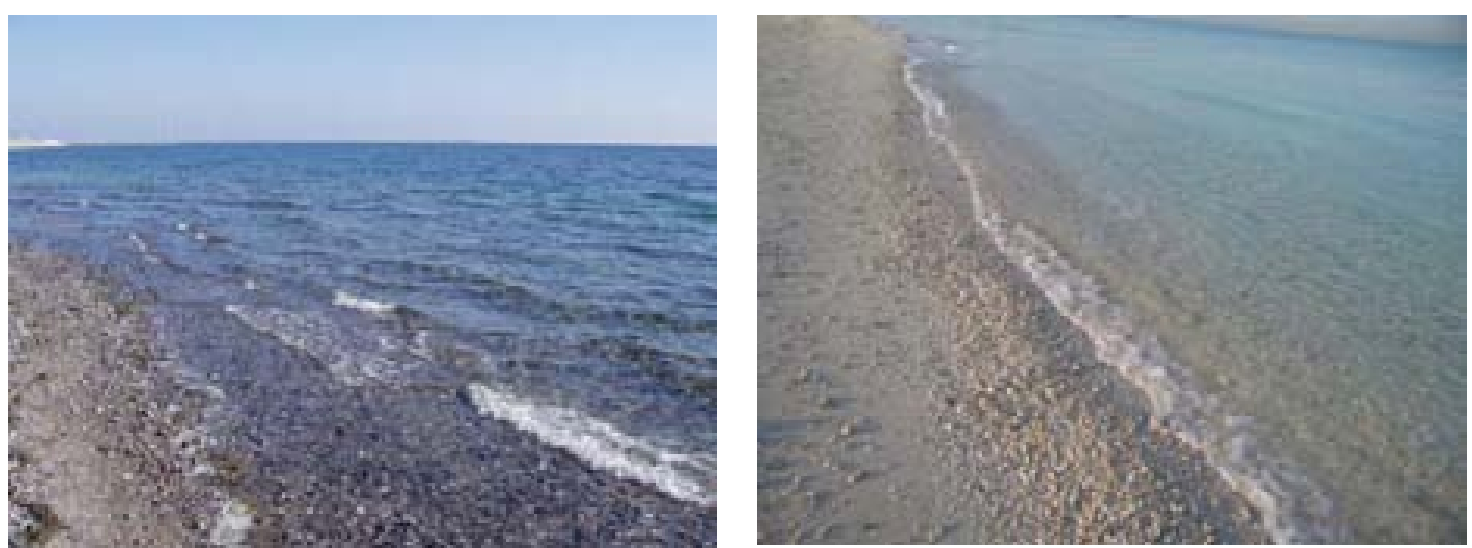

Figure 3. Plages artificielles avec un contenu de matériaux grossier trop élevé : plage avec du gravier à Amager Beach Park au Danemark (droite) et plage artificielle avec un contenu de coquillages et débris de corral, Emirats Arabes Unis (gauche). 


\section{XI ìmes Journées Nationales Génie Côtier-Génie Civil \\ Les Sables d'Olonne, 22-25 juin 2010}

\section{Exemples d'aménagement axés sur une gestion souple du littoral}

\subsection{Amager Beach Park, Danemark}

Un nouveau parc récréatif a récemment été construit à Copenhague, Danemark (voir figure 4). Celui-ci est basé sur le principe de créer de nouvelles plages exposées à la houle en les transposant vers le large afin d'éviter l'abri occasionné par la faible pente du profil de plage existant. Les directions prédominantes de la houle sur le site, NordEst et Sud-Est, ont été utilisées pour créer deux tronçons de plage séparés par une pointe rigide, l'un orienté vers le Nord-Est et l'autre orienté vers le Sud-Est.

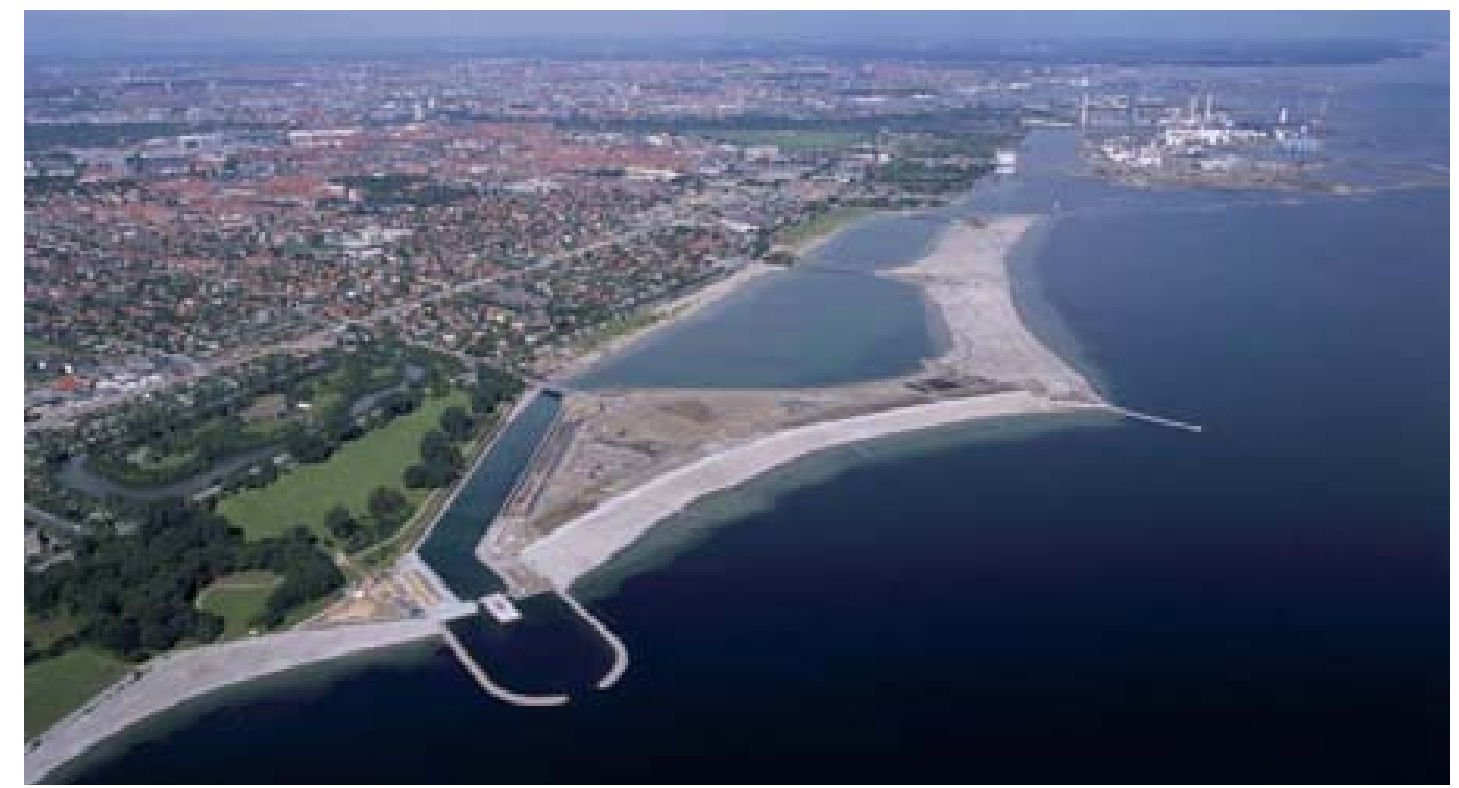

Figure 4 .Photo aérienne de Amager Beach Park à Copenhague, Danemark, prise immédiatement après sa finalisation.

\subsection{Anse de la Nourriguel, France}

La protection du mur dans le fond de l'anse de la Nourriguel à Larmor-Plage dans le Finistère (voir figure 5) fut initiée par une approche de solutions classiques d'aménagement (renforcement/épaississement du mur actuel, mise en place d'un linéaire d'enrochement, ...). La solution finalement adoptée repose sur la création d'une plage artificielle stabilisée par un épi. Cette solution consiste à amortir la houle sur la plage artificielle devant le mur et introduit également une plage récréative de bonne qualité et sans besoin d'entretien. 

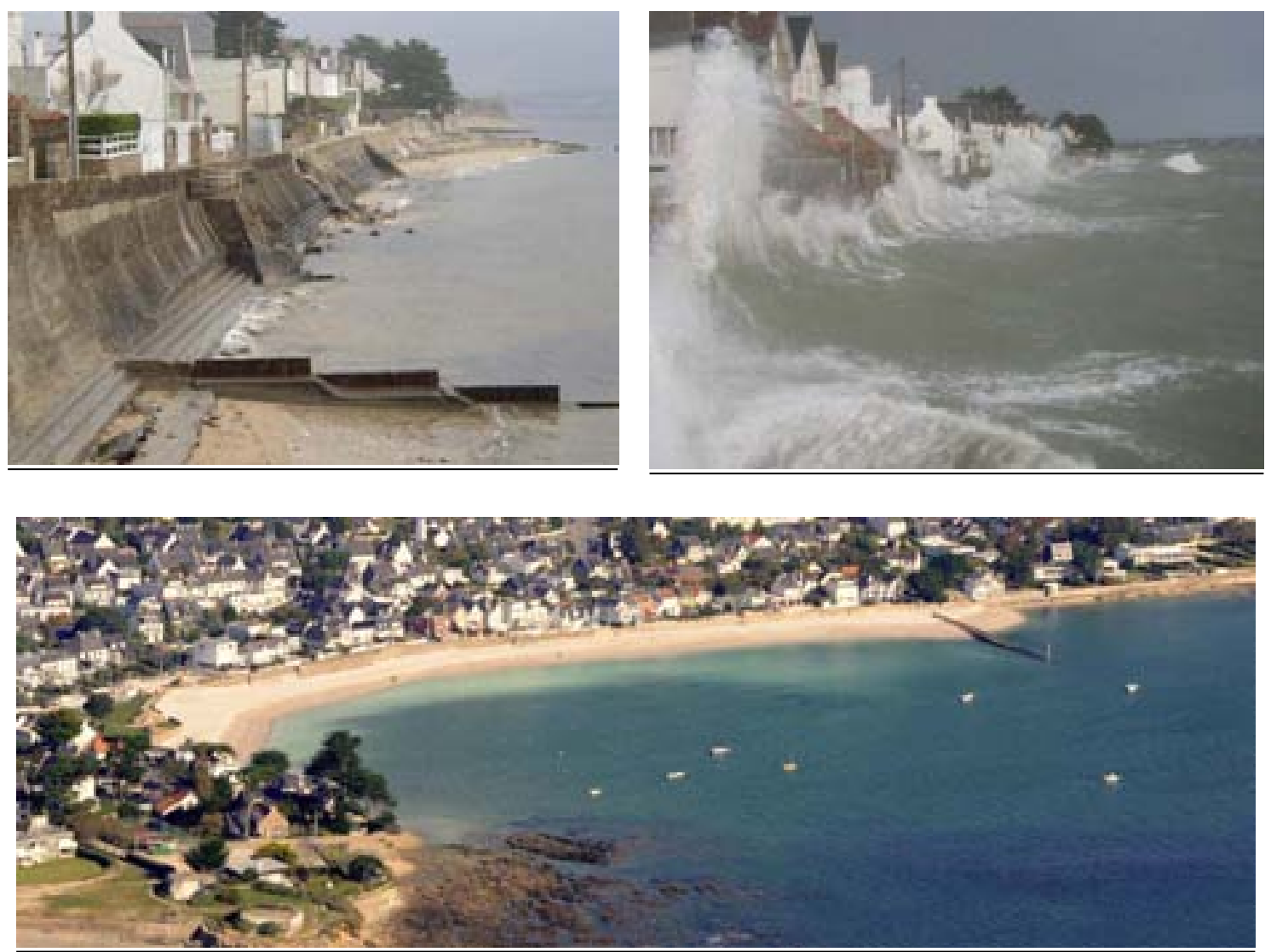

Figure 5. Situation avant (haut) et après (bas) l'aménagement d'une plage artificielle stabilisée par un épi dans le fond de l'anse de la Nourriguel, France.

\subsection{Plage et port à Alexandrie, Egypte}

Le principe d'aménager une zone partiellement abritée et de stabiliser le trait de côte sur un littoral exposé est présenté sur la figure 6. Cette figure illustre un projet d'aménagement d'une plage artificielle et d'un port à Alexandrie en Egypte. Les briselames assurent une zone abritée; la largeur de l'ouverture a été ajustée de façon à maintenir une exposition adéquate à la houle afin d'assurer une bonne qualité de la plage et de façon à obtenir des conditions de baignade en toute sécurité. La plage a été conçue de façon à être stable pour les conditions de houle prédominantes et un alignement concave résulte de la diffraction de la houle. Aucuns courants tourbillonnaires dangereux ne sont créés à cause de la distance entre les brise-lames et la plage et le profil en équilibre de la plage. 


\section{XI ìmes Journées Nationales Génie Côtier-Génie Civil}

Les Sables d'Olonne, 22-25 juin 2010
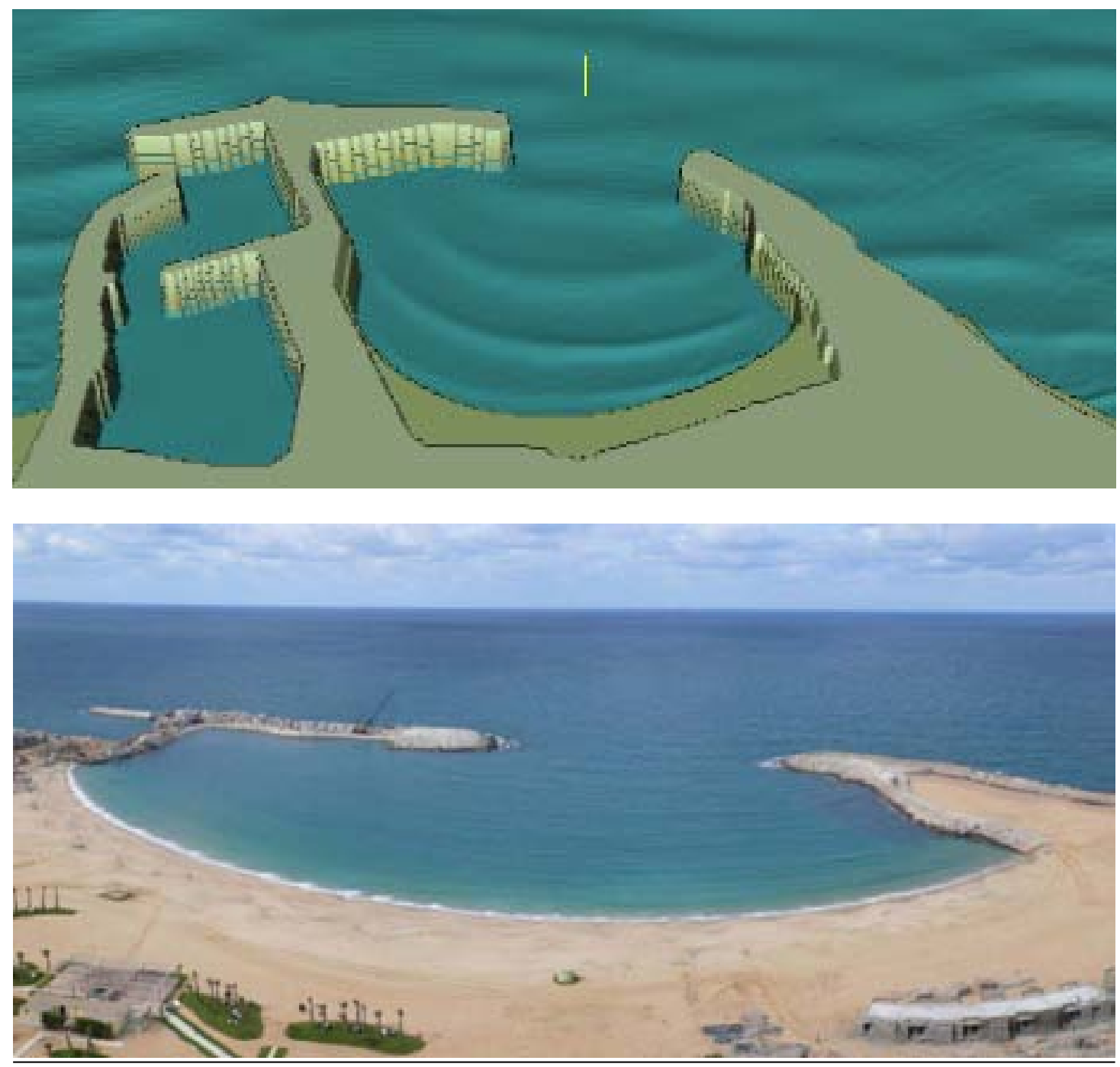

Figure 6. Modélisation de la houle (haut) avec MIKE 21 BW (DHI, 2010) et réalisation du projet d'aménagement (bas) d'une plage artificielle à Alexandrie, Egypte.

\section{Conclusions}

Pour la réussite d'un projet d'aménagement de plage artificielle, il est impératif d'inclure les aspects hydrauliques, côtiers et environnementaux dès le début du planning du projet. Le design de ces éléments doit suivre des "lois d'équilibre sédimentaire" qui imposent certaines restrictions sur le design. Les éléments les plus importants à observer sont les suivants : la plage rechargée devrait être exposée à la houle, orientée vers la direction prédominante de la houle pour être stable et soutenue par des ouvrages latéraux afin d'éviter des fuites de sédiments. Le sable devrait être moyen et bien trié avec un minimum de contenu de matériaux fins, grossiers et organiques. Les ouvrages côtiers adjacents aux plages devraient être aménagés de façon à ne pas générer des courants dangereux. 
Thème 4 - Vulnérabilité du littoral

\section{Références bibliographiques}

DEAN R.G (2003). Beach nourishment: Theory and Practice, Advances Series on Ocean Engineering - Vol. 18. World Scientific, Singapour, 420 p.

DHI (2010). Module de houle Boussinesq MIKE 21 BW de la suite du logiciel MIKE by DHI, version 2010 . 